\title{
Validación del cuestionario de Competencia Digital Docente en profesorado universitario chileno
}

\author{
Validation of the Digital Teaching Competence Questionnaire in Chilean University Teachers \\ Validação do questionário de Competência em Ensino Digital em Professores \\ Universitários Chilenos
}

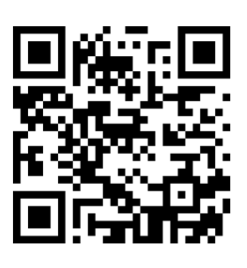

Irma Riquelme-Plaza Universidad Autónoma de Chile Santiago, Chile

irma.riquelme@uautonoma.cl https://orcid.org/0000-0002-5040-9555

Julio Cabero-Almenara

Universidad de Sevilla

Sevilla, España

cabero@us.es

https://orcid.org/0000-0002-1133-6031

Verónica Marín-Díaz

Universidad de Córdoba

Córdoba, España

ed1madiv@uco.es

https://orcid.org/0000-0001-9836-2584

Recibido • Received • Recebido: 22 / 06 / 2020

Corregido • Revised • Revisado: 25 / 10 / 2021

Aceptado • Accepted • Aprovado: 11 / 12 / 2021

\begin{abstract}
Resumen:
Objetivo. En la actualidad es clave que el profesorado universitario desarrolle competencias para entregar una educación de calidad, entre ellas, la competencia digital. El objetivo de este estudio fue validar el "Cuestionario de competencia digital docente" en la escala de uso para el contexto de la educación superior en Chile. Metodología. El método es cuantitativo, un diseño ex post facto. La muestra estuvo conformada por 1122 docentes. Resultados. Los resultados revelan que el instrumento es válido y fiable. Conclusiones. El modelo obtenido mediante análisis factorial confirmatorio indicó que el factor de mayor peso es la mantención de los dispositivos que realiza el profesorado universitario y el factor de menor peso es la utilización en la creación de contenido digital. La discusión sobre la preeminencia de la dimensión tecnológica por sobre la pedagógica busca orientar los procesos de mejora en la docencia universitaria.
\end{abstract}

Palabras clave: Análisis factorial; cuestionario; docencia universitaria; tecnologías de la información y la comunicación. 
https://doi.org/10.15359/ree.26-1.9

https://www.revistas.una.ac.cr/index.php/educare

educare@una.ac.cr

\begin{abstract}
:
Objective. Nowadays, university teachers must develop competencies to offer quality education, including digital competence. This study aimed to validate the "Teaching Digital Competence Questionnaire" in the scale of use for the context of higher education in Chile. Method. The method is quantitative, an ex-post factum study. The sample was of 1,122 teachers. Results. The results reveal that the instrument has a high level of validation and reliability. Conclusions. The model achieved through confirmatory factor analysis shows that the factor with greater weight is the maintenance of devices by the university teachers, and the factor with lesser importance is the use of devices in creating digital content. The discussion on the preeminence of the technological dimension over the pedagogical seeks to guide the improvement processes in university teaching.
\end{abstract}

Keywords: Factorial analysis; questionnaire; university teaching; information and communication technologies.

\title{
Resumo:
}

Objetivo. Atualmente, é fundamental que os professores universitários desenvolvam competências para oferecer uma educação de qualidade, incluindo a competência digital. O objetivo deste estudo foi validar o Questionário de Competência em Ensino Digital na escala de uso para o contexto do ensino superior no Chile. Metodologia. $O$ método é quantitativo, um estudo ex post facto. A amostra foi composta por 1.122 professores. Resultados. Os resultados revelam que o instrumento é válido e confiável. Conclusão. O modelo obtido pela análise fatorial confirmatória indicou que o fator de maior peso é a manutenção dos dispositivos realizados por professores universitários e o fator de menor peso é o uso na criação de conteúdo digital. A discussão sobre a preeminência da dimensão tecnológica sobre a pedagógica busca orientar os processos de melhoria no ensino universitário.

Palavras-chave: Análise fatorial; questionário; ensino universitário; tecnologia da informação e comunicação.

\section{Introducción}

Los actuales escenarios de incertidumbre en la sociedad han generado cambios en el sistema educativo $y$, en especial, en la universidad, como generadora de profesionales de alto nivel. La docencia universitaria es central en la formación de las personas para desenvolverse adecuadamente en las sociedades del siglo XXI. Esta actividad es central en los procesos de aprendizaje, por el desarrollo de las competencias que se requieren ante la complejidad e incertidumbre de las sociedades contemporáneas (Prendes Espinosa et al., 2018).

Se entenderá por competencias los conocimientos, habilidades y actitudes necesarias para adaptarse a una sociedad de rápidos cambios, inclusiva y con nuevas tecnologías (Luna Scott, 2015). En este contexto, la competencia digital es considerada una competencia clave, con un rol protagónico y transversal a todas las disciplinas (Gisbert Cervera et al., 2016). 
El actual paradigma de la educación con centralidad en el estudiantado implica cambios en el rol docente y sus competencias (Cabero Almenara y Marín Díaz, 2017). Las instituciones de educación superior no pueden estar ajenas a estos cambios, por su compromiso con la educación de calidad. En el marco de la creciente incorporación de las tecnologías de la información y la comunicación (TIC), se espera que estas instituciones promuevan el desarrollo de la competencia digital en sus estudiantes. En este escenario, son fundamentales las competencias digitales que el personal docente universitario desarrolle y promueva (Prendes Espinosa et al., 2018). El personal docente universitario debe desarrollar su competencia digital como herramienta para facilitar el aprendizaje estudiantil (Viñals Blanco y Cuenca Amigo, 2016).

\section{Competencia digital y cuerpo docente}

La competencia digital involucra múltiples definiciones que han variado en el tiempo. A modo de ejemplo, se habla de alfabetización mediática, nuevas alfabetizaciones, multimodalidad, alfabetización informática, alfabetización digital, educación en medios, alfabetización informacional, multialfabetizaciones, alfabetización TIC, e-competence, e-Skills, alfabetización tecnológica, alfabetizaciones de medios digitales y nuevos medios de información. Pese a la importancia que suscita para el proceso educativo, estudios recientes revelan la falta de un marco conceptual unánime o la omisión de este para referirse a la competencia digital (Reis et al., 2019).

En cuanto a la teorización de la competencia digital, Larraz Rada (2013) la define como la capacidad de movilizar - de forma ética y segura- cuatro alfabetizaciones: informativa, tecnológica, multimedia y comunicativa. La informativa se refiere a reconocer, localizar, evaluar, organizar y transformar la información; la tecnológica corresponde a la organización y gestión del hardware y software en distintos formatos; la multimedia en la cual se comprenden y elaboran mensajes en este formato y la comunicativa, donde se presenta y difunde la información y se participa en la ciudadanía digital.

La competencia digital es un fenómeno más amplio que el de las alfabetizaciones, ya que involucra otras dimensiones en donde se utilizan los medios digitales para comunicar, resolver, crear y construir conocimiento de manera reflexiva (Venkatesh et al., 2016). La competencia digital va más allá de lo técnico (Rodríguez-García et al., 2019) y se complejiza aún más cuando es necesario llevarla a la práctica docente, donde interactúan lo disciplinar, lo pedagógico y lo tecnológico (Gisbert Cervera et al., 2016).

En el ámbito internacional, otra de las complejidades existentes es la variedad de marcos para definir las competencias digitales docentes en los distintos niveles del sistema educativo. Al respecto, encontramos el Digital Competence Framework que es un marco amplio aplicable a todos los niveles educativos (Ferrari, 2013), el cual ha sido revisado para construir el European Framework for Digitally-Competent Educational Organisations (DIGCOMPORG). 
https://doi.org/10.15359/ree.26-1.9

https://www.revistas.una.ac.cr/index.php/educare

educare@una.ac.cr

Este último marco está centrado en los agentes educativos, pero además considera los recursos y roles de la institución. La importancia de estos marcos sobre las competencias digitales a nivel universitario reside en que consideran aspectos principalmente de índole tecnológica y pedagógica, pero incluyen también la investigación y la gestión (Prendes Espinosa et al., 2018).

EnEspaña, dondeexiste un importante desarrollo de estamateria, elMinisterio deEducación, a través del Instituto Nacional de Tecnologías Educativas y de Formación del Profesorado (INTEF), definió un "Marco común de competencia digital docente", el cual se encuentra organizado en cinco áreas: información, comunicación, creación de contenidos, seguridad y resolución de problemas (Instituto Nacional de Tecnologías Educativas y de Formación del Profesorado [INTEF], 2017; Rodríguez-García et al., 2019). En el caso de Latinoamérica, la bibliografía es diversa y de desigual profundidad, y no existe un marco común entre los países de esta región para la alfabetización digital, la adaptación de las prácticas docentes o la organización de la formación en las futuras personas pedagogas (Rivera-Laylle et al., 2017).

Considerando la situación anterior, en este estudio rescatamos el valor de la competencia digital docente (CDD) definida por el INTEF como "el uso creativo, crítico y seguro de las tecnologías de información y comunicación para alcanzar los objetivos relacionados con el trabajo, la empleabilidad, el aprendizaje, el tiempo libre, la inclusión y la participación de la sociedad" (INTEF, 2017, p. 12).

En la educación superior es importante la actualización de las competencias digitales docentes. El profesorado universitario valora positivamente las TIC en el proceso de enseñanza-aprendizaje aun cuando la integración es baja (Agreda Montoro et al., 2016). Si bien el personal docente declara la necesidad de utilizar la tecnología, no siempre cuenta con la formación suficiente, o no se encuentra completamente a su disposición para vincularla al proceso educativo. Adicionalmente, este escenario se complica cuando el estudiantado tampoco tiene las competencias digitales sólidamente desarrolladas (Agreda Montoro et al., 2016). A nivel universitario, las competencias digitales docentes se han desarrollado de manera dispar y no han respondido a las expectativas de mejoramiento en el proceso de enseñanza-aprendizaje (Mercader y Gairín, 2017), el desconocimiento del personal docente repercute en la implementación de las tecnologías, lo cual redunda en un menor uso en el espacio educativo (Almerich et al., 2016). Por otro lado, cuando el profesorado las pone en práctica, manifiesta la falta de evaluación y reflexión sobre el uso pedagógico de las tecnologías (Svensson y Baelo, 2015). Al respecto, diversos estudios reconocen la importancia del personal docente como agente de cambio, para que el estudiantado desarrolle sus competencias digitales y participe activamente en las sociedades del presente siglo (Mirete Ruiz, 2016). 
En el contexto chileno existe el marco Estándares TIC para la formación inicial docente, diseñado por Enlaces, el Centro de Educación y Tecnología del Ministerio de Educación de Chile, en colaboración con la Oficina Regional de Educación para América Latina y el Caribe (OREALC). El documento señala que la competencia digital abarca las dimensiones: pedagógica; técnica; gestión escolar; desarrollo profesional; aspectos éticos, legales y sociales. Asimismo, el organismo impulsó un marco de competencias tecnológicas para desarrollar en el sistema escolar (Enlaces, 2011).

Recientemente, se han realizado adaptaciones de instrumentos a la realidad chilena para conocer la competencia digital docente a nivel universitario (Lázaro-Cantabrana et al., 2018; Ríos Ariza et al., 2018). Sin embargo, desde nuestro conocimiento no hay estudios estadísticos multivariables para las CDD en la universidad, en muestras grandes de docentes. En función de lo anterior y de la incipiente investigación en el contexto universitario chileno, se busca aportar al desarrollo docente en esta materia, y contribuir a la formación de profesionales integrales para el siglo XXI.

\section{Metodología}

\section{Diseño}

El objetivo principal de este trabajo es adaptar y validar un instrumento para medir la competencia digital del profesorado universitario en Chile.

El estudio tiene un enfoque cuantitativo, es de tipo no experimental, de carácter exploratorio-descriptivo, cuyo alcance es transversal y tuvo un diseño ex post facto (Rodríguez Aramendiz y Gallardo Córdova, 2019).

\section{Instrumento}

Se trabajó con el Cuestionario de competencia digital docente (CDD) elaborado por Tourón et al. (2018), el cual posee dos escalas: una que mide conocimientos y otra que mide los usos referidos a la competencia digital. Este instrumento toma en consideración el Marco común de competencia digital docente establecido en España por el Ministerio de Educación (INTEF, 2017). El cuestionario consta de 5 dimensiones y 54 preguntas tipo Likert de 7 puntos (1 equivale a "nunca" y 7 a "siempre"). Para efectos de este escrito, se presentan los resultados de la escala de uso de Competencia digital docente. El cuestionario de CDD fue adaptado a la educación superior chilena, mediante modificaciones lingüísticas al contexto.

Las cinco áreas comprendidas en este Cuestionario de competencia digital se describen a continuación (ver Tabla 1): 
https://doi.org/10.15359/ree.26-1.9

https://www.revistas.una.ac.cr/index.php/educare

educare@una.ac.cr

Tabla 1: Áreas del marco común de competencia digital docente 2017

\begin{tabular}{|c|c|}
\hline Área & Descripción General \\
\hline $\begin{array}{l}\text { Información y alfabetización } \\
\text { informacional. }\end{array}$ & $\begin{array}{l}\text { Identificar, localizar, obtener, almacenar, organizar y analizar información digital, } \\
\text { datos y contenidos digitales, evaluando su finalidad y relevancia para las tareas } \\
\text { docentes (INTEF, 2017, p. 15). }\end{array}$ \\
\hline $\begin{array}{l}\text { Comunicación y } \\
\text { colaboración. }\end{array}$ & $\begin{array}{l}\text { Comunicar en entornos digitales, compartir recursos a través de herramientas en } \\
\text { línea, conectar y colaborar con otros a través de herramientas digitales, interactuar } \\
\text { y participar en comunidades y redes; conciencia intercultural (INTEF, 2017, p. 23). }\end{array}$ \\
\hline Creación de contenido digital. & $\begin{array}{l}\text { Crear y editar contenidos digitales nuevos, integrar y reelaborar conocimientos } \\
\text { y contenidos previos, realizar producciones artísticas, contenidos multimedia y } \\
\text { programación informática, saber aplicar los derechos de propiedad intelectual y } \\
\text { las licencias de uso (INTEF, 2017, p. 37). }\end{array}$ \\
\hline Seguridad. & $\begin{array}{l}\text { Protección de información y datos personales, protección de la identidad digital, } \\
\text { protección de los contenidos digitales, medidas de seguridad y uso responsable y } \\
\text { seguro de la tecnología (INTEF, 2017, p.47). }\end{array}$ \\
\hline Resolución de problemas. & $\begin{array}{l}\text { Identificar necesidades de uso de recursos digitales, tomar decisiones informadas } \\
\text { sobre las herramientas digitales más apropiadas según el propósito o la necesidad, } \\
\text { resolver problemas conceptuales a través de medios digitales, usar las tecnologías } \\
\text { de forma creativa, resolver problemas técnicos, actualizar su propia competencia } \\
\text { y la de otros (INTEF, 2017, p. 57). }\end{array}$ \\
\hline
\end{tabular}

Nota: Elaboración propia a partir de INTEF (2017).

\section{Muestra}

El personal docente participante de esta investigación pertenecen a la UniversidadTecnológica de Chile (INACAP), que junto al Instituto Profesional (IP) y Centro de Formación Técnica (CFT) con el mismo nombre, forman parte de una corporación de derecho privado, sin fines de lucro. Su modelo curricular considera un alto uso de tecnologías. Esta universidad privada cuenta con 26 sedes en todo Chile, en las regiones de zona norte, centro y sur y cubre una amplia oferta disciplinar: administración y negocios, agropecuaria y agroindustrial, construcción, diseño y comunicación, electricidad y electrónica, hotelería, turismo y gastronomía, humanidades y educación, informática y telecomunicaciones, mecánica, minería y metalurgia, procesos industriales y salud.

La muestra es de tipo no probabilística. Las personas participantes del estudio se seleccionaron mediante un muestreo incidental por accesibilidad (Marín-Díaz et al., 2017). La muestra total estuvo conformada por 1122 docentes, siendo $31,8 \%$ mujeres y $68,2 \%$ del personal docente son hombres y en el caso de carreras técnicas y de ingeniería el 97\% son varones (Consejo Nacional de Educación [CNED], s. f.). En cuanto a la experiencia laboral, la mayoría $(28,8 \%)$ tiene entre 6 y 10 años de ejercicio como docente y el tramo etario predominante en esta muestra es entre los 41 y 50 años (33,5\%). 


\section{Procedimiento de levantamiento y análisis de datos}

Los cuestionarios fueron administrados mediante un formulario online generado en formsite. Para su implementación se solicitó el permiso correspondiente a las autoridades presentes en las sedes de la Universidad, y se respondieron consultas del personal docente telefónicamente y por correo electrónico sobre el estudio. En todo momento se les informó del anonimato y confidencialidad de la participación, cuyos datos serían de uso exclusivo para esta investigación. Los datos fueron recabados entre mayo y julio del año 2018.

\section{Plan de análisis}

Para la validación del cuestionario se realizaron estudios de fiabilidad y validez, con el objetivo de que el instrumento y la información obtenida tuviera la suficiente rigurosidad científica. Los datos del estudio fueron sometidos a análisis de confiabilidad, mediante la aplicación del coeficiente alfa de Cronbach, cuyos valores, si son cercanos a 1, indican una alta confiabilidad en las escalas (Marín-Díaz, et al., 2017).

Para determinar la validez de constructo del instrumento, se realizó primeramente un análisis factorial exploratorio (AFE) con el objeto de detectar los factores latentes, los que fueron un insumo para el posterior análisis factorial confirmatorio (AFC) (Hair et al., 2014).

Los programas utilizados para el análisis de datos fue el Statistical Package for the Social Sciences (SPSS) v. 23, y en específico, el módulo Analysis of Moment Structures Graphics.

\section{Resultados}

La consistencia interna para la escala de uso de la competencia digital docente obtuvo un alfa de Cronbach de .979, lo que indica una alta confiabilidad por lo que no se eliminó ningún ítem.

En el AFE se consideraron los ítems cuyas cargas (pesos) fuesen igual o mayor a .60 (60\% de la varianza explicada) con el objetivo de disminuir la cantidad de variables que componen cada factor (Hair et al., 2014).

En cuanto a la bondad de ajuste del AFE se obtiene un KMO de .954, muy cercano a 1 lo que indica una relación alta de los ítems entre sí. Los valores obtenidos con la prueba de esfericidad de Bartlett señalan que es posible realizar un análisis factorial (Esfericidad de Bartlett $=15048.380 ; p=0.000$ ).

De este primer análisis es posible extraer 4 factores que explican el 69,964\% de la varianza total. El método de extracción fue de componentes principales con rotación Varimax, del cual se obtiene la siguiente matriz de componentes rotados (ver Tabla 2). 
https://doi.org/10.15359/ree.26-1.9

https://www.revistas.una.ac.cr/index.php/educare

educare@una.ac.cr

Tabla 2: Matriz de componente rotado para el grado de uso

\begin{tabular}{ccccc}
\hline & \multicolumn{3}{c}{ Componente } \\
\cline { 2 - 4 } & 1 & 2 & 3 & 4 \\
\hline
\end{tabular}

Cómo mantener una actitud equilibrada en el uso de la tecnología.

.757

Opciones para combinar tecnología digital y no digital para buscar soluciones. $\quad .723$

Formas para controlar el uso de la tecnología que se convierten en aspectos $\quad .694$ distractores.

Criterios para evaluar la fiabilidad de las fuentes de información, datos, contenido $\quad .692$ digital, etc.

Recursos digitales adaptados al proyecto educativo [de la sede].

Actividades didácticas creativas desarrollar la competencia digital en el alumnado. $\quad .681$

Herramientas para reelaborar o enriquecer contenido en diferentes formatos (p. .662 ej.: textos, tablas, audio, imágenes, vídeos, etc.).

Espacios para compartir archivos, imágenes, trabajos, etc.

El software de la pizarra digital interactiva de mi [sede].

Herramientas de contenido basado en realidad aumentada.

Herramientas para crear grabaciones de voz (podcast).

Formas de gestión de identidades digitales en el contexto educativo.

Herramientas para producir códigos QR (Quick Response).

Soluciones para la gestión y el almacenamiento en la «nube», compartir archivos, concesión de privilegios de acceso, etc. (... Drive, OneDrive, Dropbox u otras).

Herramientas para el almacenamiento y gestión de archivos y contenidos compartidos (... Drive, Box, Dropbox, Office 365, etc.).

Sistemas de protección de dispositivos o documentos (control de acceso, privilegios, contraseñas, etc.).

Estrategias de gestión de la información (empleo de marcadores, recuperación de información, clasificación, etc.).

Tareas básicas de mantenimiento del [computador] para evitar posibles problemas de funcionamiento ... (actualizaciones, limpieza de caché o de disco, etc.).

Formas para eliminar datos/información de la que es responsable sobre sí o la de terceras personas.

Protección para ... dispositivos de amenazas de virus, malware, etc.

Nota: Elaboración propia a partir de Tourón et al. (2018, pp.50-52). 
Para la estimación del AFC, se realizó el ajuste global del modelo con los siguientes estadísticos: Chi cuadrado ( $\left.\mathrm{X}^{2}\right)$, índice de bondad de ajuste (GFI), índice de bondad de ajuste corregido (AGFI), índice de ajuste comparativo (CFI) y error de aproximación cuadrático medio (RMSEA). Las "medidas oscilan entre 0 (mal ajuste) a 1.0 (ajuste perfecto) Los ajustes ... con valores superiores a .90 ... se consideran aceptables" (Montenegro Maggio y González Ugalde, 2013, p. 221).

El estadístico Chi cuadrado $\left(X^{2}=896.415, g l=164, p=.00\right)$, muestra un buen ajuste del modelo según rechazo de hipótesis nula, esto debido a que la prueba es sensible al tamaño muestral. En el ajuste de medida del modelo, la relación que existe entre cada ítem y factor es significativo, ya que las cargas de cada ítem tienen un valor de lambda sobre 0,5 (MorataRamírez et al., 2015).

RMSEA representa el valor que podría esperarse si el modelo fuera estimado con la población y no solamente con la muestra extraída. Para efectos de significancia, los valores que van de .05 a .08 se consideran aceptables (Hair et al., 2014, p. 221). La raíz cuadrada del error cuadrático medio (RMSEA) es de .063 con un intervalo de confianza del 90\% (.059 y .067) lo cual, está dentro de valores aceptables (Montenegro Maggio y González Ugalde, 2013).

El índice de bondad de ajuste GFI adquiere un valor de .886; el índice de bondad de ajuste corregido AGFI es de .854; y el índice de ajuste comparativo CFI es de .737, todos valores son próximos a 1, por lo tanto, el modelo es de calidad aceptable. En el modelo propuesto, y según los pesos obtenidos se obtienen los siguientes factores:

Factor 1 (peso: 3.48) se ha llamado "mantenimiento", aunque en el modelo final propuesto por Tourón et al. (2018) aparece como subdimensión. Para el contexto chileno, este es el factor que tiene el mayor peso. La mayor saturación de sus ítems corresponde a la realización de tareas básicas para proteger los datos.

Factor 2 (peso: 2,60) está conformado por ocho ítems, cuatro corresponden a "problemas", dos a "creación digital", uno a "gestión de la información" y otro a "comunicación". Considerando esta agrupación este factor se denomina "problemas", de la competencia digital docente, donde destacan los ítems lograr mantener una actitud equilibrada en el uso de la tecnología y la opción para combinar tecnología digital y no digital para buscar soluciones.

Factor 3 (peso: 2.53) se ha denominado "gestión de la información", puesto que todos los ítems agrupados forman parte de esa dimensión en el modelo final obtenido por Tourón et al. (2018). En este factor los ítems de mayor peso, corresponden al uso de sistemas de protección de dispositivos y de documentos.

Factor 4 (peso: 2,07) está compuesto por cinco ítems, cuatro corresponden a creación digital y uno a comunicación. Este factor se ha denominado creación digital y obtienen mayor 
https://doi.org/10.15359/ree.26-1.9

https://www.revistas.una.ac.cr/index.php/educare

educare@una.ac.cr

saturación los ítems sobre el uso de software tales como la pizarra digital, la realidad aumentada y las grabaciones de voz. En la Figura 1, se ilustra el modelo.

Figura 1: Modelo para el grado de uso

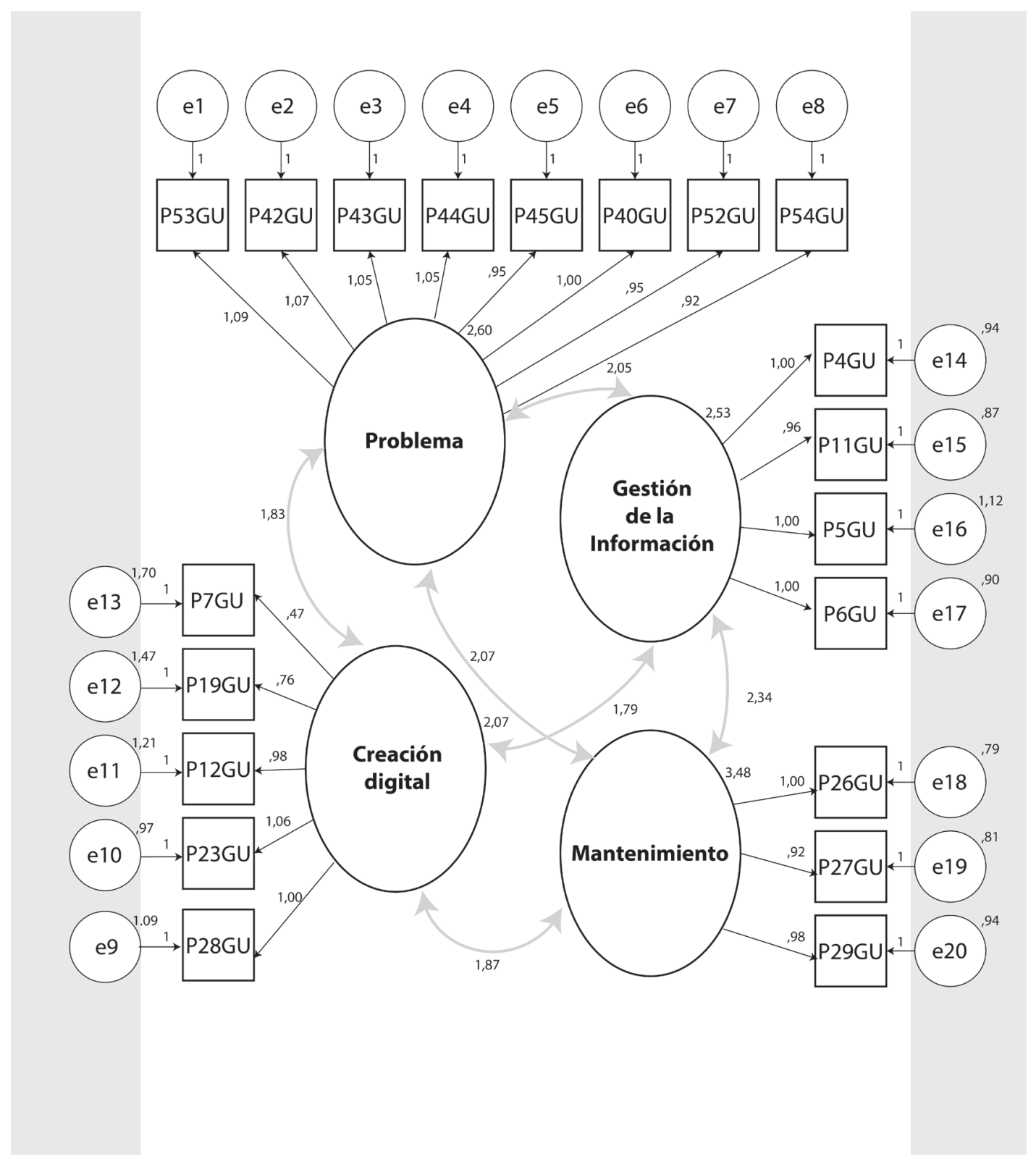

Nota: Elaboración propia. 
En cuanto a las relaciones entre estos factores, la relación de mayor peso está entre gestión de la información y mantenimiento $(2,34)$. enseguida, se posiciona la relación entre problema y gestión de la información $(2,05)$. La relación de menor peso está entre creación de contenido digital y gestión de la información $(1,79)$.

En términos comparativos, para el contexto chileno todas las relaciones entre los factores del modelo elaborado presentan un peso mayor peso que en el estudio sobre el país ibérico. En contraste con el escenario español, no aparece el factor de comunicación y colaboración, lo que coincidiría con estudios recientes que señalan que en el personal docente universitario todavía no hay suficiente dominio en el aspecto colaborativo (Mercader y Gairín, 2017).

\section{Discusiones y conclusiones}

El objetivo de este estudio fue adaptar y validar el "Cuestionario de competencia digital docente (CDD)" diseñado en el contexto de España, para conocer el uso de estas competencias en la educación superior en Chile. Esta investigación constituye una aproximación cuantitativa inédita que considera a más de mil docentes, de las regiones de este país, pertenecientes a diversas unidades académicas y disciplinares.

El alfa de Cronbach obtenido respalda la alta confiabilidad del cuestionario. El análisis factorial exploratorio arrojó cuatro factores latentes, que posteriormente se demuestran con el análisis factorial confirmatorio, mediante un modelo que presenta resultados consistentes y válidos. Adicionalmente, los coeficientes obtenidos muestran una mayor saturación que en el modelo español.

Las dimensiones que se obtienen sobre el uso de competencias digitales docentes fueron: i) mantenimiento, ii) problemas, iii) gestión de la información, y iv) creación digital. Esto difiere con el modelo propuesto por Tourón et al. (2018), quienes plantean los factores: gestión de la información, problemas, creación digital y comunicación. la dimensión mantenimiento para el caso chileno constituye una subdimensión para el país europeo dentro del factor problemas, lo que advierte la preeminencia de los aspectos técnicos por sobre la creación de contenidos digitales para uso pedagógicos en Chile.

La metodología utilizada para la validación del instrumento puede ser ampliada en otros países y niveles del sistema educativo. Cabe señalar que el instrumento se aplicó a través de formsite, plataforma de fácil acceso para el personal docente ubicado en distintas zonas geográficas y, además, facilitó la recolección de datos para su posterior análisis. Sin embargo, el uso de la competencia digital se sitúa en una perspectiva declarativa, puesto que la recopilación de información se basa en lo que los sujetos señalan (autopercepción) y no en lo que realmente implementan (Podsakoff et al., 2012). 
https://doi.org/10.15359/ree.26-1.9

https://www.revistas.una.ac.cr/index.php/educare

educare@una.ac.cr

La competencia digital es un constructo complejo en cuya conceptualización se identifican perspectivas divergentes, y en reiteradas ocasiones, la homologación con la alfabetización digital que provoca ambigüedad y referencias cruzadas (Reis et al., 2019). Por tanto, estamos frente a un concepto en desarrollo que carece de una definición clara y única (Rodríguez-García, et al., 2019; Tourón et al., 2018).

En Chile todavía no existe un marco común que defina la CDD en el contexto universitario, y que esté vinculado explícitamente al perfil docente. Por esta razón, avanzar en su conceptualización es relevante para sentar las bases de la docencia universitaria de calidad. El desarrollo de esta competencia en el personal docente universitario permite crear nuevos entornos de aprendizaje y promover el desarrollo de la competencia digital en el estudiantado (Cabero Almenara y Marín Díaz, 2017; Mirete Ruiz, 2016; Tondeur et al., 2018).

Este estudio busca ser un aporte para el fortalecimiento de la competencia digital docente en Chile, ofreciendo insumos para orientar los esfuerzos institucionales de las universidades en cuanto a la formación del personal docente (Prendes Espinosa et al., 2018). De esta manera, se plantea una necesidad formativa de carácter holística, que potencie el desarrollo de la competencia digital en los diversos ámbitos del quehacer académico, y con especial énfasis en las dimensiones de creación digital y gestión de la información, aquellas en las que el personal docente declaró un menor uso (Mirete Ruiz, 2016). La integración entre la tecnología, la disciplina y la pedagogía en el profesorado universitario es compleja, ya que hay una desconexión entre estos tres elementos, lo cual es relevante en el proceso de formación docente (Chua Reyes et al., 2017).

Asimismo, se plantea la inclusión de las dimensiones obtenidas en los procesos de certificación de las competencias en el personal docente de educación superior. La percepción que tenga el personal docente sobre sus propias competencias podría ser complementada con demostraciones de desempeño. Si bien en la realidad española existen proyectos al respecto, en el caso de Chile todavía en el nivel universitario es incipiente (Lázaro-Cantabrana et al., 2018; Ríos Ariza et al., 2018). Esto permitiría abrir nuevas líneas de trabajo, en la cual se intencione el ámbito pedagógico del uso creativo, crítico y seguro de las tecnologías de información y comunicación (Gisbert Cervera et al., 2016).

Futuros estudios podrían complementar la información que el personal docente declara en el cuestionario con aquella sobre el uso efectivo de la competencia digital docente en el espacio de aprendizaje, para comprender mejor este fenómeno. También sería interesante indagar si el desarrollo de esta competencia es coherente con el enfoque de centralidad en el estudiantado, ampliando la investigación sobre la práctica docente en esta materia.

El valor de la competencia digital tiene relación con la adquisición de conocimientos que utilizan las tecnologías y herramientas de comunicación digitales, el desarrollo de competencias sociales e interculturales para la interacción eficaz con otras personas -muchas veces a distancia- 
y la participación responsable y ética en la ciudadanía digital y en las comunidades virtuales (Luna Scott, 2015). De esta manera, la importancia del desarrollo de esta competencia en la educación superior trasciende las posibilidades de aprendizaje, y remite a la participación en un mundo cada vez más interconectado.

Finalmente, se espera contar con nuevos resultados y perspectivas para profundizar en la competencia digital del profesorado universitario en Chile, y su relación e impacto en el desarrollo de la competencia digital en sus estudiantes, participantes de la actual ciudadanía digital y de la economía del conocimiento impulsada por la tecnología.

\section{Declaración de Material complementario}

Este artículo tiene disponible, como material complementario:

-La versión preprint del artículo en https://doi.org/10.5281/zenodo.5517873

\section{Referencias}

Agreda Montoro, M., Hinojo Lucena, M. A. y Sola Reche, J. M. (2016). Diseño y validación de un instrumento para evaluar la competencia digital de los docentes en la educación superior española. Píxel-Bit. Revista de Medios y Educación, (49), 39-56. https://doi.org/10.12795/ pixelbit.2016.i49.03

Almerich, G., Orellana, N., Suárez-Rodríguez, J. y Díaz-García, I. (2016). Teachers' information and communication technology competences: A structural approach. Computers and Education, 100, 110-125. https://doi.org/10.1016/j.compedu.2016.05.002

Cabero Almenara, J. y Marín Díaz, V. (2017). La educación formal de los formadores de la era digital - los educadores del siglo XXI. Notandum, (44-45), 29-42. http://dx.doi.org/10.4025/ notandum.44.4

Chua Reyes, Jr., V., Reading, C., Doyle, H.y Gregory, S. (2017). Integrating ICT into teacher education programs form a TPACK perspective: Exploring perceptions of university lecturers. Computers \& Education, 115, 1-19. https://doi.org/10.1016/j.compedu.2017.07.009

Consejo Nacional de Educación. (s. f.). Índices indicadores educación superior. https://www.cned. cl/indices

Enlaces. (2011). Marco de competencias tecnológicas para el sistema escolar. http://www.enlaces. cl/marco-de-competencias-tecnologicas-para-el-sistema-escolar/

Ferrari, A. (2013). DIGCOMP: A framework for developing and understanding digital competence in Europe. European Union. 10.2788/52966 
https://doi.org/10.15359/ree.26-1.9

https://www.revistas.una.ac.cr/index.php/educare

educare@una.ac.cr

Gisbert Cervera, M., González Martínez, J. y Esteve Mon, F. (2016). Competencia digital y competencia digital docente: Una panorámica sobre el estado de la cuestión. RIITE. Revista Interuniversitaria de Investigación en Tecnología Educativa, (0), 74-83. http://dx.doi. org/10.6018/riite/2016/257631

Hair, J. F., Black, W. C., Babin, B. J. y Anderson, R. E. (2014). Multivariate data analysis: Pearson new international edition. Pearson.

Instituto Nacional de Tecnologías Educativas y de Formación del Profesorado. (2017). Marco común de competencia digital docente. https://aprende.intef.es/sites/default/ files/2018-05/2017 1020 Marco-Com\%C3\%BAn-de-Competencia-Digital-Docente.pdf

Larraz Rada, V. (2013). La competència digital a la universitat [Tesis doctoral]. Universitat d'Andorra. http://hdl.handle.net/10803/113431

Lázaro-Cantabrana, J. L., Gisbert-Cervera, M. y Silva-Quiroz, J. E. (2018). Una rúbrica para evaluar la competencia digital del profesor universitario en el contexto latinoamericano. EDUTEC. Revista Electrónica de Tecnología Educativa, (63), 1-14. https://doi.org/10.21556/ edutec.2018.63.1091

Luna Scott, C. (2015). El futuro del aprendizaje 2. ¿Qué tipo de aprendizaje se necesita en el siglo XXI? (Documentos de Trabajo ERF). Investigación y Prospectivas en Educación UNESCO, (14), 1-14. https://www.uees.edu.sv/wp-content/uploads/2017/planeamiento/doc/El\%20 futuro\%20del\%20aprendizaje.pdf

Marín-Díaz, V., Sampedro Requena, B. E. y Vega Gea, E. (2017). Estudio psicométrico de la aplicación del internet addiction test con estudiantes universitarios españoles. Contextos educativos, Extraordinario 2, 147-161. https://doi.org/10.18172/con.3067

Mercader, C. y Gairín, J. (2017). ¿Cómo utiliza el profesorado universitario las tecnologías digitales en sus aulas? REDU. Revista de Docencia Universitaria, 15(2), 257-273. https://doi. org/10.4995/redu.2017.7635

Mirete Ruiz, A. B. (2016). El profesorado universitario y las TIC. Análisis de su competencia digital. ENSAYOS, Revista de la Facultad de Educación de Albacete, 31(1), 133-147. https:// doi.org/10.18239/ensayos.v31i1.1033

Montenegro Maggio, H. y González Ugalde, C. (2013). Análisis factorial confirmatorio del cuestionario "Enfoques de docencia universitaria". Estudios Pedagógicos, 39(2), 213-230. https://doi.org/10.4067/S0718-07052013000200014

Morata-Ramírez, M. A., Holgado-Tello, F. P., Barbero-García, I. y Méndez, G. (2015). Análisis factorial confirmatorio. Recomendaciones sobre mínimos cuadrados no ponderados en función del error Tipo I de Ji cuadrado y RMSEA. Acción Psicológica, 12(1), 79-90. https:// scielo.isciii.es/scielo.php?script=sci arttext\&pid=S1578-908X2015000100008

Podsakoff, P. M., MacKenzie, S. B. y Podsakoff, N. P. (2012). Sources of method bias in social science research and recommendations on how to control it. Annual Review of Psychology, 63, 539569. https://doi.org/10.1146/annurev-psych-120710-100452 
Prendes Espinosa, M. P., Gutiérrez Porlán, I. y Martínez Sánchez, F. (2018). Competencia digital una necesidad del profesorado universitario en el siglo XXI. RED. Revista de Educación a Distancia, (56), 1-22. http://dx.doi.org/10.6018/red/56/7

Reis, C., Pessoa, T. y Gallego-Arrufat, M. J. (2019). Alfabetización y competencia digital en Educación Superior: Una revisión sistemática. REDU. Revista de Docencia Universitaria, 17(1), 45-58. https://doi.org/10.4995/redu.2019.11274

Ríos Ariza, J. M., Gómez Barajas, E. R., y Rojas Polanco, M. P. (2018). Valoración de competencias TIC del profesorado universitario: Un caso en Chile. Píxel-Bit. Revista de Medios y Educación, (52), 55-65. https://doi.org/10.12795/pixelbit.2018.i52.04

Rivera-Laylle, L. I., Fernández-Morales, K., Guzmán-Games, F. J. y Eduardo-Pulido, J. (2017). La aceptación de las TIC por profesorado universitario: Conocimiento, actitud y practicidad. Revista Electrónica Educare, 21(3), 1-18. https://doi.org/10.15359/ree.21-3.6

Rodríguez Aramendiz, V. R. y Gallardo Córdova, K. E. (2019). Decisiones en evaluación: Ambientes virtuales de posgrado, un estudio ex post-facto. RED. Revista de Educación a Distancia, 19 (59), 1-19. https://doi.org/10.6018/red/59/06

Rodríguez-García, A.-M., Raso Sánchez, F. y Ruiz-Palmero, J. (2019). Competencia digital, educación superior y formación del profesorado: Un estudio de meta-análisis en la web of science, Píxel-Bit. Revista de Medios y Educación, (54), 65-81. https://doi.org/10.12795/ pixelbit.2019.i54.04

Svensson, M. y Baelo, R. (2015). Teacher students' perceptions of their digital competence. Procedia. Social and Behavioral Sciences, 180, 1527-1534. https://doi.org/10.1016/j. sbspro.2015.02.302

Tondeur, J., Aesaert, K., Prestridge, S. y Consuegra, E. (2018). A multilevel analysis of what matters in the training of pre-service teacher's ICT competencies. Computers \& Education, 122, 3242. https://doi.org/10.1016/j.compedu.2018.03.002

Tourón, J, Martín, D., Navarro Asencio, E., Pradas, S. e Iñigo, V. (2018). Validación de constructo de un instrumento para medir la competencia digital docente de los profesores (CDD). REP. Revista Española de Pedagogía, 76(269), 25-54. https://doi.org/10.22550/REP76-1-2018-02

Venkatesh, V., Rabah, J., Fusaro, M., Couture, A., Varela, W. y Alexander, K. (2016). Factors Impacting University Instructors' and Students' Perceptions of Course Effectiveness and Technology Integration in the Age of Web 2.0. McGill Journal of Education, 51(1), 533-561. https://doi. org/10.7202/1037358ar

Viñals Blanco, A. y Cuenca Amigo, J. (2016). El rol del docente en la era digital. Revista Interuniversitaria de Formación del Profesorado, 30(2), 103-114.https://www.redalyc.org/ jatsRepo/274/27447325008/html/index.html 